\title{
DIFFERENTIAL PROPERTIES \\ OF LIPSCHITZ, HAMILTONIAN AND CHARACTERISTIC FLOWS
}

\author{
Stefan Mirica \\ Faculty of Mathematics, University of Bucharest \\ Academiei 14, 70109 Bucharest, Romania \\ mirica@math.math.unibuc.ro
}

\begin{abstract}
In view of possible applications to Hamilton-Jacobi equations, to optimal control and to differential games, we extend the classical differential properties of smooth Hamiltonian and Characteristic flows to Lipschitzean ones using the contingent derivatives of their components
\end{abstract}

Keywords: Hamiltonian system and flow, Characteristic system and flow, Lipschitzean mapping, contingent derivative

Mathematics Subject Classification: 34A12; 49J52; 35B37

\section{Introduction}

The aim of this paper is to generalize in terms of the contingent derivatives the basic differential relation:

$$
D V(t, z) \cdot(r, u)=<P(t, z), D X(t, z) \cdot(r, u)>-r \cdot H\left(t, X^{*}(t, z)\right)-<q, u_{1}>
$$

if $z=(\xi, q), u=\left(u_{1}, u_{2}\right)$, satisfied by the components of a smooth Characteristic flow $C^{*}(.,):.=\left(X^{*}(.,),. V(.,).\right)$, that is uniquely associated to a smooth Hamiltonian system:

$$
\left(x^{\prime}, p^{\prime}\right)=h(t, x, p):=\left(\frac{\partial H}{\partial p}(t, x, p),-\frac{\partial H}{\partial x}(t, x, p)\right),(x(T), p(T))=z \in D^{T}
$$

in the following way: the (smooth) Hamiltonian flow $X^{*}(.,):.=(X(.,),. P(.,)$.$) :$ $D_{h} \subseteq R \times D^{T} \rightarrow R^{n} \times R^{n}$ is defined by the unique, maximal (i.e. non-continuable) solutions $X^{*}(., z): I(z) \subseteq R \rightarrow R^{n} \times R^{n}, z \in D^{T}:=\{(\xi, q) ;(T, \xi, q) \in D\}$ of the problem (1.2) while the third component is given by the formula:

$$
V(t, z):=\int_{T}^{t}\left[<P(s, z), \frac{\partial H}{\partial p}\left(s, X^{*}(s, z)\right)>-H\left(s, X^{*}(s, z)\right)\right] d s .
$$

This type of relations are essential for the construction of classical and generalized "characteristic solutions" of Hamilton-Jacobi equations, for deriving "Hopf-Lax formulas" and are particularly useful in optimal control and differential games.

The original version of this chapter was revised: The copyright line was incorrect. This has been corrected. The Erratum to this chapter is available at DOI: 10.1007/978-0-387-35690-7_44 
In this paper the Hamiltonian $H(., . .):. D=\operatorname{Int}(D) \subseteq R \times R^{n} \times R^{n} \rightarrow R$ is differentiable with respect to the last two variables and such that the corresponding Hamiltonian vector field $h(., .,$.$) in (1.2) is a "Carathéodory-Lipschitz" mapping; un-$ der these hypotheses, if $T \in p r_{1} D$ then the unique maximal Characteritic flow $C^{*}(.,$. is locally-Lipschitz with respect to the second variable and locally- $A C$ (absolutely continuous) with respect to the first one so the basic relation in (1.1) does not make sense any more.

The main result of this paper, Theorem 3.4 below, states that in this case the contingent derivatives of the components $X(.,),. V(.,$.$) satisfy certain relations that$ coincide with the one in (1.1) in the particular case $h(., .,$.$) is a smooth (Hamilto-$ nian) vector field; for the proof of this result we essentially use the generalization in Blagodatskih(1973) and Mirică(1985,2002) of the Bendixson-Picard-Lindelöf theorem on differentiability of solutions with respect to initial data in the theory of ODE; this type of proof is new and apparently simpler than the traditional proofs of the relation (1.1) in the classical case (e.g. Courant(1962), Hartman(1964), Mirică(1987) etc).

The paper is organized as follows: in Section 2 we present the necessary notations, definitions and preliminary results from Nonsmooth Analysis and from the theory of Carathéodory differential equations and in Section 3 we present the main results.

\section{Notations, definitions and preliminary results}

From the multitude of the existing concepts in Nonsmoth Analysis (e.g. Aubin and Frankowska(1990), Mirică(1982), etc.), we shall use in the first place the setvalued contingent directional derivative of a mapping $f():. X \subseteq R^{n} \rightarrow R^{k}$ at a point $x \in \operatorname{Int}(X)$ in a direction $u \in R^{n}$ defined by:

$$
K^{+} f(x ; u):=\left\{v \in R^{k} ; \exists\left(s_{m}, u_{m}\right) \rightarrow\left(0_{+}, u\right): \frac{f\left(x+s_{m} u_{m}\right)-f(x)}{s_{m}} \rightarrow v\right\}
$$

and, in the case $g():. X \subseteq R^{n} \rightarrow R$ is a real function we may use also its extreme contingent derivatives (to the right) at $x \in \operatorname{Int}(X)$ in direction $u \in R^{n}$ :

$$
\begin{aligned}
& \bar{D}_{K}^{+} g(x ; u):=\limsup _{(s, v) \rightarrow\left(0_{+}, u\right)} \frac{g(x+s . v)-g(x)}{s}, \\
& \underline{D}_{K}^{+} g(x ; u):=\liminf _{(s, v) \rightarrow\left(0_{+}, u\right)} \frac{g(x+s . v)-g(x)}{s}
\end{aligned}
$$

In what follows we shall consider only the particular case in which the domain $X=$ $\operatorname{dom}(f())=.\operatorname{dom}(g().) \subseteq R^{n}$ is open and the mappings $f(),. g($.$) are locally-Lipschitz.$

We recall first that for this type of mappings the contingent derivatives in (2.1), (2.2) have the properties in the following Proposition whose proof is straightforward (see Aubin and Frankowska(1990), Mirică(1982), etc.):

Proposition 2.1. If the mapping $f():. X=\operatorname{Int}(X) \subseteq R^{n} \rightarrow R^{k}$ is locally-Lipschiz and $x \in X$ then its contingent derivative in (2.1) has the following properties:

(i) in any direction $u \in R^{n}$ the subset $K^{+} f(x ; u) \subset R^{k}$ is non-empty and compact and is given by:

$$
K^{+} f(x ; u):=\left\{v \in R^{k} ; \exists s_{m} \rightarrow 0_{+}: \frac{f\left(x+s_{m} u\right)-f(x)}{s_{m}} \rightarrow v\right\}
$$

(ii) the multifunction $K^{+} f(x ;$.$) is ("globally") Lipschitzean with respect to the$ Pompeiu-Hausdorff distance in the sense that:

$$
d_{H}\left(K^{+} f(x ; u), K^{+} f\left(x ; u^{\prime}\right)\right) \leq L .\left\|u-u^{\prime}\right\| \forall u, u^{\prime} \in R^{n}
$$


where $L$ is the Lipschitz constant of $f($.$) at x$ and:

$$
d_{H}(A, B):=\max \left\{d^{*}(A, B), d^{*}(B, A)\right\}, d^{*}(A, B):=\sup _{a \in A} \inf _{b \in B}\|a-b\| .
$$

Moreover, $K^{+} f(x ;$.$) is positively homogeneous in the sense that:$

$$
K^{+} f(x ; \lambda . u)=\lambda . K^{+} f(x ; u) \forall u \in R^{n}, \lambda \geq 0, K^{+} f(x ; 0)=\{0\} ;
$$

(iii) the mapping $f($.$) is (Fréchet) differentiable at the point x \in X$ iff it is contingent differentiable in any direction $u \in R^{n}$ in the sense that:

$$
\exists f_{K}^{+}(x ; u):=\lim _{(s, v) \rightarrow\left(0_{+}, u\right)} \frac{f(x+s . v)-f(x)}{s} \forall u \in R^{n}
$$

and the "contingent derivative" $f_{K}^{+}(x ;):. R^{n} \rightarrow R^{k}$ is linear; in this case the two derivatives coincide i.e. $D f(x)=f_{K}^{+}(x ;$.$) .$

(iv) if $g():. X \rightarrow R$ is locally-Lipschitz then its contingent derivatives in (2.1)-(2.2) are related as follows:

$$
\bar{D}_{K}^{+} g(x ; u)=\max \left[K^{+} g(x ; u)\right] \geq \min \left[K^{+} g(x ; u)\right]=\underline{D}_{K}^{+} g(x ; u) \forall u \in R^{n} .
$$

Remark 2.2. According to the well-known Rademacher's theorem, the locallyLipschitz mapping $f($.$) in Prop.2.1 is a.e. differentiable hence there exists a subset$ $\mathcal{D}_{F}(f) \subseteq X$, of "full Lebesgue measure" $(\mu()$.$) such that:$

$$
\exists D f(x)=f_{K}^{+}(x ; .) \forall x \in \mathcal{D}_{F}(f), \mu(X \backslash \mathcal{D}(f))=0 ;
$$

on the other hand, as simple examples show, the set of points at which the contingent derivative in (2.6) exists may be strictly larger than the (Fréchet) "differentiablity set" in (2.7):

$$
\mathcal{D}_{F}(f) \subseteq \mathcal{D}_{K}^{+}(f):=\left\{x \in X ; \operatorname{dom}\left(f_{K}^{+}(x ; .)\right)=R^{n}\right\} ;
$$

equivalently, for each point $x \in X$ one may consider the "set of contingent differentiability directions" of $f($.$) at x$ defined as the domain of the mapping $f_{K}^{+}(x ;$.$) :$

$$
\mathcal{D}_{K}^{+}(f ; x):=\operatorname{dom}\left(f_{K}^{+}(x ; .)\right):=\left\{u \in R^{n} ; \exists f_{K}^{+}(x ; u)\right\}
$$

so that one may write: $\mathcal{D}_{K}^{+}(f)=\left\{x \in X ; \mathcal{D}_{K}^{+}(f ; x)=R^{n}\right\}$.

In this paper we shall use these concepts and results to the study of the "maximal" (i.e. non-continuable) flow, $x_{f}(. ; .,):. D_{f} \subseteq R \times D \rightarrow R^{n}$, of a Carathéodory-Lipschitz differential equation:

$$
x^{\prime}=f(t, x), x(s)=y,(s, y) \in D=\operatorname{dom}(f(., .)) \subseteq R \times R^{n}
$$

defined, more precisely, as follows:

Definition 2.3. A mapping $f(.,):. D \rightarrow R^{n}$ is said to be a Carathéodory-Lipschitz (C-L) vector field if $D \subset R \times R^{n}$ is open and the following properties hold:

(i) $f(.,$.$) is a Carathéodory mapping in the sense that the mappings f(., x), x \in$ $p r_{2} D$ are (Lebesgue) measurable, there exists a null subset $I_{f} \subset p r_{1} D$ (i.e. $\mu\left(I_{f}\right)=0$ ) such that $f(t,),. t \in p r_{1} D \backslash I_{f}$ are continuous and, moreover, $f(.,$.$) is locally integrably$ bounded in the sense that for any compact subset $D_{0} \subset D$ there exist an integrable function $m(.) \in L^{1}\left(p r_{1} D_{0} ; R_{+}\right), R_{+}=[0, \infty)$ and a null subset $I_{0} \subset p r_{1} D_{0}$ such that:

$$
\|f(t, x)\| \leq m(t) \forall(t, x) \in D_{0}, t \in p r_{1} D_{0} \backslash I_{0} ;
$$


(ii) $f(.,$.$) is locally-integrably Lipschitz with respect to the second variable in the$ sense that for any compact subset $D_{0} \subset D$ there exist an integrable function $L(.) \in$ $L^{1}\left(p r_{1} D_{0} ; R_{+}\right)$and a null subset $I_{0} \subset p r_{1} D_{0}$ such that:

$$
\|f(t, x)-f(t, y)\| \leq L(t)\|x-y\| \forall(t, x),(t, y) \in D_{0}, t \in p r_{1} D_{0} \backslash I_{0} .
$$

Remark 2.4. As particular cases of the C-L vector fields in Def. 2.3 one may consider the locally-essentially bounded ones when the function $m($.$) in (2.11)$ is essentialy bounded (i.e. $\left.m(.) \in L^{\infty}\left(p r_{1} D_{0} ; R_{+}\right)\right)$, the essentially bounded locally-Lipchitz ones for which $L(.) \in L^{\infty}\left(p r_{1} D_{0} ; R_{+}\right)$and the Carathéodory- $C^{1}$ vector fields for which, in addition to the properties in Def.2.3, one assumes that the mappings $f(t,),. t \in$ $p r_{1} D \backslash I_{f}$ are differentiable and, moreover, the derivative, $D_{2} f(.,$.$) is of Carathéodory$ type i.e. $D_{2} f(., x), x \in p r_{2} D$ are measurable, the mappings $D_{2} f(t,),. t \in p r_{1} D \backslash I_{f}$ are continuous and for any compact subset $D_{0} \subset D$ there exist $L(.) \in L^{1}\left(p r_{1} D_{0} ; R_{+}\right)$ and a null subset $I_{0} \subset p r_{1} D_{0}$ such that:

$$
\left\|D_{2} f(t, x)\right\| \leq L(t) \forall(t, x),(t, y) \in D_{0}, t \in p r_{1} D_{0} \backslash I_{0} .
$$

As further particular cases one may consider Peano-Lipschitz vector fields $f(.,$.$) wich$ are continuous with respect to both variables and locally-Lipschitz with respect to the second variable, uniformly with respect to the first one (i.e. the function $L($.$) in$ (2.12) is constant) and the "classical" Peano- $C^{1}$ vector fields for which both mappings $f(.,),. D_{2} f(.,$.$) are continuous.$

We summerize the basic results of the theory of Carathéodory Ordinary Differential Equations in the following theorem for whose proof we refer to Kurzweil(1986), Ch.18: Theorem 2.5. If $f(.,$.$) is a Carathéodory-Lipschitz (C-L) vector field in the sense$ of Def.2.3 then the following statements hold:

(i) for any initial point $(s, y) \in D$ there exists a unique maximal (i.e. noncontinuable) locally-AC (absolutely continuous on each compact interval) Carathéodory solution $x_{f}(. ; s, y): I(s, y) \subset R \rightarrow R^{n}$ of the equation in (2.10) that satisfies:

$$
D_{1} x_{f}(t ; s, y)=f\left(t, x_{f}(t ; s, y)\right) \text { a.e. }(t \in I(s, y)), x_{f}(s ; s, y)=y ;
$$

(ii) moreover, $I(s, y)$ is an open interval containing $s$, the domain of the flow, $D_{f}=\{(t, s, y) ;(s, y) \in D, t \in I(s, y)\} \subseteq R \times D$ is an open subset, and the "maximal flow" $x_{f}(. ; .,):. D_{f} \rightarrow R^{n}$ is continuous;

(iii) the mappings $x_{f}(t ; s,$.$) are locally Lipschitz and the mappings x_{f}(. ; s, y)$, $x_{f}(t ; ., y)$ are locally- $A C$ and satisfy the equivalent "associated integral equation"

$$
x_{f}(t ; s, y)=y+\int_{s}^{t} f\left(\sigma, x_{f}(\sigma ; s, y)\right) d \sigma \forall(t, s, y) \in D_{f} .
$$

Therefore, each of the mappings, $x_{f}(. ; s, y),(s, y) \in D$ satisfies the equation (2.10) outside of a null subset of the interval $I(s, y)$; however, the following important result shows that equation (2.10) is satisfied outside a "common" null subset by all the solutions:

Theorem 2.6 (Scorza-Dragoni(1948)). If $f(.,$.$) is a C-L vector field in the sense$ of Def.2.3 then there exists a null subset $J_{f} \subset p r_{1} D$ such that:

$$
D_{1} x_{f}(t ; s, y)=f\left(t, x_{f}(t ; s, y)\right) \forall t \in I(s, y) \backslash J_{f},(s, y) \in D .
$$

For a proof of this theorem (actually valid for the more general class of the Carathéodory vector fields in Def.2.3) one may see also Th.18.4.9 in Kurzweil (1986). 
The differentiability properties of the flow $x_{f}(. ; .,$.$) in Th.2.5 will be expressed, as$ usual, along a (fixed) "reference trajectory", $z($.$) , given by:$

$$
z(t)=x_{f}\left(t ; t_{0}, x_{0}\right), t \in I\left(t_{0}, x_{0}\right)
$$

where $\left(t_{0}, x_{0}\right) \in D$ is a fixed point, in terms of the set-valued contingent directional derivatives in (2.1) of the mappings $f(t,),. x_{f}\left(t ; t_{0},.\right), x_{f}\left(t ; ., x_{0}\right)$ and of the Carathéodory solutions, $v($.$) , of the contingent variational inclusion (CVI):$

$$
v^{\prime}(t) \in c o\left[K^{+} f(t ; .)(z(t) ; v(t))\right] \text { a.e. }\left(I\left(t_{0}, x_{0}\right)\right), v\left(t_{0}\right)=u_{0} \in R^{n}
$$

where $\operatorname{co}[A]$ denotes the convex hull of the subset $A \subset R^{n}$ which, as it is well-known, coincides with the closed convex hull, $\overline{c o}[A]$, whenever $A$ is compact; using the "contingent differentiability directions" in (2.9) one may see that at certain points the CVI in (2.18) becomes the contingent variational equation (CVE):

$$
\left.v^{\prime}(t)=(f(t ; .))_{K}^{+}(z(t) ; v(t)) \text { a.e. }\left(I\left(t_{0}, x_{0}\right)\right) \text {, if } v(t) \in \mathcal{D}_{K}^{+}(f(t ; .) ; z(t))\right),
$$

while on the ("full measure") Fréchet differentiability sets in (2.7) the CVI in (2.18) becomes the classical variational equation (VE):

$$
v^{\prime}(t)=D_{2} f(t, z(t)) \cdot v(t) \text { a.e. }\left(I\left(t_{0}, x_{0}\right)\right) \text {, if } z(t) \in \mathcal{D}_{F}(f(t, .))
$$

In the proof of the main result in the next section we shall essentially uses the following generalization of the classical Bendixson-Picard- Lindelöf theorem:

Theorem 2.7. Let $f(.,):. D \rightarrow R^{n}$ be a Carathéodory- Lipschitz vector field in the sense of Def.2.3, let $\left.\left(t_{0}, x_{0}\right)\right) \in D, t_{1} \in I\left(t_{0}, x_{0}\right), I_{1}:=\left[t_{0}, t_{1}\right] \subset I\left(t_{0}, x_{0}\right)$ and let $z($. be the reference trajectory in (2.17).

Then the contingent derivatives in (2.1) of the maximal flow $x_{f}(. ; .,$.$) in Th.2.5$ have the following properties:

(i) for any vectors $u_{0} \in R^{n}, u_{1} \in K^{+} x_{f}\left(t_{1} ; t_{0},.\right)\left(x_{0} ; u_{0}\right)$ there exists a Carathéodory solution $v():. I_{1} \rightarrow R^{n}$ of the contingent variational inclusion CVI such that:

$$
v\left(t_{0}\right)=u_{0}, v\left(t_{1}\right)=u_{1}, v(t) \in K^{+} x_{f}\left(t ; t_{0}, .\right)\left(x_{0} ; u_{0}\right) \forall t \in I_{1}
$$

(ii) if $J_{f} \subset p r_{1} D$ is the null subset in (2.16) and $t_{0} \in p r_{1} D \backslash J_{f}$ then for any vector $u_{1}^{0} \in K^{+} x_{f}\left(t_{1} ; ., x_{0}\right)\left(t_{0} ; 1\right)$ there exists a Carathéodory solution $v^{0}($.$) of the contingent$ variational inclusion (CVI) in (2.18) such that:

$$
v^{0}\left(t_{0}\right)=-f\left(t_{0}, x_{0}\right), v^{0}\left(t_{1}\right)=u_{1}^{0}, v^{0}(t) \in K^{+} x_{f}\left(t ; ., x_{0}\right)\left(t_{0} ; 1\right) \forall t \in I_{1} .
$$

Moreover, if $f(.,$.$) is locally essentially bounded in the sense of Remark 2.4$ and $t_{0} \in J_{f}$ then for any vector $u_{1}^{0} \in K^{+} x_{f}\left(t_{1} ; ., x_{0}\right)\left(t_{0} ; 1\right)$ there exists a Carathéodory solution $v^{0}($.$) of the contingent variational inclusion CVI in (2.18) that satisfies the last two$ conditions in (2.22) and also the "weaker" initial condition:

$$
\left.v^{0}\left(t_{0}\right) \in-f^{c o}\left(t_{0}+, x_{0}\right), f^{c o}(s+, y):=\bigcap_{\delta>0} \bigcap_{\mu(J)=0} \overline{c o} f(([s, s+\delta) \backslash J)) \times B_{\delta}(y)\right) .
$$

For a proof of this result we refer to Blagodatskih(1973) and Mirică(1985,2002). 


\section{The main results}

Everywhere in what follows we assume the following:

Hypothesis 3.1. The Hamiltonian $H(., .,):. D=\operatorname{Int}(D) \subseteq R \times R^{n} \times R^{n} \rightarrow R$ is such that there exists a null subset, $I_{H} \subset p r_{1} D$ (i.e. $\mu\left(I_{H}\right)=0$ ) such that the functions $H(t, .,),. t \in \operatorname{pr}_{1} D \backslash I_{H}$ are (Fréchet) differentiable and such that the corresponding Hamiltonian vector field $h(., .,)=.\left(h_{1}(., .,),. h_{2}(., .,).\right)$ in (1.2) is a CarathéodoryLipschitz vector field in the sense of Def.2.3; moreover, the null subset $I_{H} \subset p r_{1} D$ is taken such that for each $t \in p r_{1} D \backslash I_{H}$ the mapping $h(t,$.$) is locally-Lipschitz (on the$ "section" $\left.D^{t}:=\left\{z \in R^{2 n} ;(t, z) \in D\right\}\right)$.

To simplify the exposition we consider here only the particular case in which the initial values of the "time-variable" in (1.2) are fixed though statement (ii) in Th.2.7 suggests the possibility of studying also the more general case in which these values are variable; noting first that the vector field of the characteristics defined by:

$$
\begin{aligned}
& c(t, x, p):=\left(h_{1}(t, x, p), h_{2}(t, x, p), c_{3}(t, x, p)\right), h_{1}(t, x, p):=\frac{\partial H}{\partial p}(t, x, p), \\
& h_{2}(t, x, p):=-\frac{\partial H}{\partial x}(t, x, p), c_{3}(t, x, p):=<p, h_{1}(t, x, p)>-H(t, x, p)
\end{aligned}
$$

is also of Carathéodory-Lipschitz type, applying Ths.2.5, 2.6 one obtains:

Theorem 3.2. If Hypothesis 3.1 is satisfied, $T \in p r_{1} D$ and $D^{T}:=\{(\xi, q) \in$ $\left.R^{n} \times R^{n} ;(T, \xi, q) \in D\right\}$ then there exists a unique characteristic flow $C^{*}(.,)=$. $\left(X^{*}(.,),. V(.,).\right): D_{h} \subseteq R \times D^{T} \rightarrow R^{n} \times R^{n} \times R$ and a null subset $J_{c} \subset p r_{1} D,\left(I_{H} \subseteq\right.$ $\left.J_{c}\right)$ such that for each $\bar{z}=(\xi, q) \in D^{T}$ the mapping $C^{*}(., z): I(z) \subseteq R \rightarrow R^{n} \times R^{n} \times R$ is the unique maximal Carathéodory solution of the "system of characteristics":

$$
\left(x^{\prime}, p^{\prime}, v^{\prime}\right)=c(t, x, p),(x(T), p(T), v(T))=(\xi, q, 0) \in D^{T} \times\{0\}
$$

satisfying:

$$
D_{1} C^{*}(t, z)=c\left(t, X^{*}(t, z)\right) \forall t \in I(z) \backslash J_{c}, C^{*}(T, z)=(z, 0), z \in D^{T} .
$$

Moreover, the characteristic flow $C^{*}(.,$.$) has the regularity properties in Th.2.5 i.e.$ the intervals $I(z) \subseteq R$ and the domain $D_{h}:=\left\{(t, z) ; z \in D^{T}, t \in I(z)\right\}$ are open, $C^{*}(.,$.$) is continuous, the mappings C^{*}(t,$.$) are Lipschitzean and the mappings C^{*}(., z)$ are locally-AC.

In addition, $X^{*}(.,)=.(X(.,),. P(.,)$.$) is the unique maximal flow in the same sense$ of the Hamiltonian system in (1.2) and the third component, $V(.,$.$) , is given by the$ formula in (1.3).

Before applying Th.2.7 to the characteristic flow above we prove first a very specific property of the contingent derivatives in (2.1) of the characteristic vector field in (3.1). Proposition 3.3. If $I_{H} \subset p r_{1} D$ is the null subset in Hypothesis 3.1 then at any point $(t, z) \in D, t \in \operatorname{pr}_{1} D \backslash I_{H}, z=(x, p) \in D^{t}$ and in any direction $u=\left(u_{1}, u_{2}\right) \in R^{2 n}$, the set-valued contingent derivative in (2.1) of the characteristic vector field in (3.1) is given by:

$$
\begin{aligned}
& K^{+} c(t, .)(z ; u)=\left\{v=\left(v_{1}, v_{2}, v_{3}\right) ;\left(v_{1}, v_{2}\right) \in K^{+} h(t, .)(z ; u),\right. \\
& \left.v_{3}=<p, v_{1}>+<h_{2}(t, z), u_{1}>\right\} \text { if } z=(x, p) \in D^{t}, t \in p r_{1} D \backslash I_{H}
\end{aligned}
$$

and its (closed) convex hull is given by:

$$
\begin{aligned}
& c o\left[K^{+} c(t, .)(z ; u)\right]=\left\{v=\left(v_{1}, v_{2}, v_{3}\right) ;\left(v_{1}, v_{2}\right) \in c o\left[K^{+} h(t, .)(z ; u)\right]\right. \\
& \left.v_{3}=<p, v_{1}>+<h_{2}(t, z), u_{1}>\right\} .
\end{aligned}
$$


where $h=\left(h_{1}, h_{2}\right)$ is the Hamiltonian vector field in (3.1).

In particular, at the Fréchet differentiability points $z=(x, p) \in \mathcal{D}_{F}(h(t,)$.$) in (2.7)$, the component $c_{3}(t,$.$) is also differentiable and:$

$$
D_{2} c_{3}(t, z) \cdot u=<p, D_{2} h_{1}(t, z) \cdot u>+<h_{2}(t, z), u_{1}>\forall u=\left(u_{1}, u_{2}\right) \in R^{n} \times R^{n} .
$$

Proof. From the equivalent definition in (2.3) (for locally-Lipschitz mappings) it follows that if $v=\left(v_{1}, v_{2}, v_{3}\right) \in K^{+} c(t,).(z ; u)$ then there exists a sequence $s_{m} \rightarrow 0_{+}$ such that:

$$
\left(v_{1}, v_{2}\right)=\lim _{m \rightarrow \infty} \frac{h\left(t, z+s_{m} u\right)-h(t, z)}{s_{m}}, v_{3}=\lim _{m \rightarrow \infty} \frac{c_{3}\left(t, z+s_{m} u\right)-c_{3}(t, z)}{s_{m}}
$$

hence $\left(v_{1}, v_{2}\right) \in K^{+} h(t,).(z ; u)$; on the other hand, from (3.6), (3.1) it follows:

$$
\begin{aligned}
v_{3}=\lim _{m \rightarrow \infty}\left[<p, \frac{h_{1}\left(t, z+s_{m} u\right)-h_{1}(t, z)}{s_{m}}>\right. & +<u_{2}, h_{1}\left(t, z+s_{h} u\right)>- \\
& \left.-\frac{H\left(t, z+s_{m} u\right)-H(t, z)}{s_{m}}\right]
\end{aligned}
$$

and since $H(t,$.$) is differentiable, from (3.1) is follows:$

$$
\lim _{m \rightarrow \infty} \frac{H\left(t, z+s_{m} u\right)-H(t, z)}{s_{m}}=-<h_{2}(t, z), u_{1}>+<h_{1}(t, z), u_{2}>
$$

hence $v_{3}=<p, v_{1}>+<u_{2}, h_{1}(t, z)>+<h_{2}(t, z), u_{1}>-<h_{1}(t, z), u_{2}>$ and the first inclusion (" $\subseteq$ ") in (3.3) is proved.

To prove the reversed inclusion we consider $v=\left(v_{1}, v_{2}, v_{3}\right)$ such that $\left(v_{1}, v_{2}\right) \in$ $K^{+} h(t,).(z ; u), v_{3}=<p, v_{1}>+<h_{2}(t, z), u_{1}>$ and note that from (2.3) it follows the existence of a sequence $s_{m} \rightarrow 0_{+}$such that the first relation in (3.6) is verified; next, since the component $c_{3}(t,$.$) in (3.1)$ is obviously locally-Lipschitz, the sequence

$$
\left\{\frac{c_{3}\left(t, z+s_{m} u\right)-c_{3}(t, z)}{s_{m}}, m \in N\right\} \subset R
$$

is bounded hence it has a convergent subsequence and therefore there exists $\bar{v}_{3} \in R$ such that, taking possibly a subsequence, one has:

$$
\left(v_{1}, v_{2}, \bar{v}_{3}\right)=\lim _{m \rightarrow \infty} \frac{c\left(t, z+s_{m} u\right)-c(t, z)}{s_{m}} \in K^{+} c(t, .)(z ; u) .
$$

Finally, from the proof above it follows that in this case $\bar{v}_{3}=<p, v_{1}>+$ $<h_{2}(t, z), u_{1}>=v_{3}$ and the relation in (3.3) is proved.

The relation in (3.4) follows, obviously from the one in (3.3) since $v_{3}$ depends "linearly" on $v_{1}$ while, in view of Prop.2.1, (3.5) is a particular case of (3.3),(3.4).

We note that the relation in (3.4), which seems to be ignored in the classical theory, may be extended in the same form to the "sets of contingent differentiable directions" $\left.u \in \mathcal{D}_{K}^{+}(h(t,)) ; z.\right)$ defined in $(2.9)$.

The main result of this paper is the following:

Theorem 3.4. If Hypothesis 3.1 is satisfied and $C^{*}(.,)=.(X(.,),. P(.,),. V(.,)$.$) is$ the characteristic flow in Th.3.2 then at each point $(t, z) \in D_{h}, z=(\xi, q) \in D^{T}$ and any direction $u=\left(u_{1}, u_{2}\right) \in R^{n} \times R^{n}$, the contingent derivatives in (2.1) with respect to the second variable of its components are related as follows:

$$
K^{+} V(t, .)(z ; u)=\left\{<P(t, z), v_{1}>-<q, u_{1}>; v_{1} \in K^{+} X(t ; .)(z ; u)\right\}
$$


and therefore the extreme contingent derivatives in (2.2), (2.6) are given by:

$$
\begin{aligned}
& \bar{D}_{K}^{+} V(t, .)(z ; u)=\max \left\{<P(t, z), v_{1}>-<q, u_{1}>; v_{1} \in K^{+} X(t ; .)(z ; u)\right\} \\
& \underline{D}_{K}^{+} V(t, .)(z ; u)=\min \left\{<P(t, z), v_{1}>-<q, u_{1}>; v_{1} \in K^{+} X(t ; .)(z ; u)\right\}
\end{aligned}
$$

In particular, if $u \in \mathcal{D}_{K}^{+}(X(t,) ; z$.$) (i.e. X(t,$.$) is contingent differentiable at z$ in direction $u$ ) then $u \in \mathcal{D}_{K}^{+}(V(t,) ; z$.$) and:$

$$
(V(t, .))_{K}^{+}(z ; u)=<P(t, z),(X(t ; .))_{K}^{+}(z ; u)>-<q, u_{1}>\text { if } u=\left(u_{1}, u_{2}\right)
$$

and if $z \in \mathcal{D}_{F}(X(t,)$.$) (i.e. X(t,$.$) is differentiable at z$ ) then $V(t,$.$) is also differen-$ tiable at $z$ and:

$$
D_{2} V(t, z) \cdot u=<P(t, z), D_{2} X(t, z) \cdot u>-<q, u_{1}>\forall u=\left(u_{1}, u_{2}\right) \in R^{2 n} .
$$

Proof. To prove the inclusion " $\subseteq$ " in (3.7) we consider $v_{3} \in K^{+} V(t,).(z ; u)$ and note that from (2.3) it follows that there exists $s_{m} \rightarrow 0_{+}$such that:

$$
v_{3}=\lim _{m \rightarrow \infty} \frac{V\left(t, z+s_{m} u\right)-V(t, z)}{s_{m}} ;
$$

next, since $C^{*}(t,)=.(X(t,),. P(t,),. V(t,)$.$) is locally-Lipschitz, as in the case above$ it follows that there exists $\left(v_{1}, v_{2}\right) \in K^{+} X^{*}(t,).(z ; u)$ such that, taking possibly a subsequence, the relations in (3.6) hold.

In order to prove that in this case one has $v_{3}=<P(t, z), v_{1}>-<q, u_{1}>$ we apply Th.2.7 to the C-L "standard" vector field defined by:

$$
\widetilde{c}(t, x, p, v):=c(t, x, p) \forall(t, x, p) \in D, v \in R
$$

and to its corresponding maximal flow, $\widetilde{C}^{*}(.,$.$) for which one obviously has:$

$$
\widetilde{C}^{*}(t, \widetilde{z})=C^{*}(t, z) \text { if } \widetilde{z}=(z, 0) \in D^{T} \times\{0\} .
$$

We take the "reference trajectory"

$$
C(s):=(X(s), P(s), V(s)):=\widetilde{C}^{*}(s, \widetilde{z})=C^{*}(s, z), s \in I_{1}=[T, t]
$$

and note that from statement (i) of Th.2.7 it follows that for the vectors $\widetilde{u}:=$ $(u, 0) \in R^{2 n} \times\{0\}, v \in K^{+} \widetilde{C}^{*}(t,).(\widetilde{z} ; \widetilde{u})$ there exists a Carathéodory solution $w()=$. $\left(w_{1}(),. w_{2}(),. w_{3}().\right) \in A C\left(I ; R^{2 n+1}\right)$ of the CVI:

$$
w^{\prime}(s) \in c o\left[K^{+} \widetilde{c}(s, .)(C(s) ; w(s))\right] \text { a.e. }\left(I_{1}\right)
$$

such that:

$$
w(T)=\widetilde{u}, w(t)=v, w(s) \in K^{+} \widetilde{C}^{*}(s, .)(\widetilde{z} ; \widetilde{u}) \forall s \in I_{1}=[T, t] .
$$

We note that from (3.12) and (2.1) it follows that:

$$
K^{+} \widetilde{c}(t, .)(\widetilde{z}, \widetilde{u})=K^{+} c(t, .)(z ; u) \text { if } \widetilde{z}=(z, r) \in D^{t} \times R, \widetilde{u}=(u, 0) \in R^{2 n} \times\{0\}
$$

hence the CVI in (3.15) becomes:

$$
w^{\prime}(s) \in c o\left[K^{+} c(s, .)\left(X^{*}(s) ;\left(w_{1}(s), w_{2}(s)\right)\right)\right] \text { a.e. }\left(I_{1}\right), X^{*}(.)=(X(.), P(.))
$$


We apply now Prop.3.3 to conclude that one has:

$$
\begin{aligned}
& \left(w_{1}^{\prime}(s), w_{2}^{\prime}(s)\right) \in c o\left[K^{+} h(s, .)\left(X^{*}(s) ; w_{1}(s), w_{2}(s)\right) \text { a.e. }\left(I_{1}\right)\right. \\
& w_{3}^{\prime}(s)=<P(s), w_{1}^{\prime}(s)>+<h_{2}\left(s, X^{*}(s)\right), w_{1}(s)>\text { a.e. }\left(I_{1}\right) .
\end{aligned}
$$

Therefore, since from (1.2) it follows that $P^{\prime}(s)=h_{2}\left(s, X^{*}(s)\right)$ a.e. $\left(I_{1}\right)$, from the last relation in (3.17) it follows that:

$$
w_{3}^{\prime}(s)=\frac{d}{d s}\left[<P(s), w_{1}(s)>\right] \text { a.e. }\left(I_{1}\right)
$$

hence using the Leibnitz-Newton formula for $A C$ mappings one obtains: $w_{3}(t)=$ $w_{3}(T)+<P(t), w_{1}(t)>-<P(T), w_{1}(T)>$ which, together with the end-point conditions in (3.16) and the fact that $X^{*}(T)=(\xi, q)=z$, proves the fact that $v_{3}=<P(t, z), v_{1}>-<q, u_{1}>$ and the first inclusion in (3.7) is proved.

To prove the reversed inclusion we consider $v_{1} \in K^{+} X(t,).(z ; u), v_{3}:=<P(t, z)$, $v_{1}>-\left\langle q, u_{1}>\right.$ and note that from (2.3) it follows that there exists $s_{m} \rightarrow 0_{+}$ such that: $v_{1}=\lim _{m \rightarrow \infty}\left(X\left(t, z+s_{m} u\right)-X(t, z)\right) / s_{m}$; as in the other cases above, since $V(t,$.$) is locally-Lipschitz it follows that there exists \bar{v}_{3} \in R$ such that, taking possibly a subsequence one has:

$$
\bar{v}_{3}=\lim _{m \rightarrow \infty} \frac{V\left(t, z+s_{m} u\right)-V(t, z)}{s_{m}} \in K^{+} V(t, .)(z ; u)
$$

hence from the proof above it follows that $\bar{v}_{3}=<P(t, z), v_{1}>-<q, u_{1}>=v_{3}$ and the theorem is completely proved since the relations in (3.9) and (3.10) are obvious particular cases of the one in (3.7).

Noting that for $r=0$ the relation in (1.1) coincide with (3.10), we prove now a more complete generalization of (1.1) in the case $r \in R$ :

Corollary 3.5. If Hypothesis 3.1 is satisfied and $J_{c} \subset p r_{1} D$ is the null subset in (3.2) then at each point $z=(\xi, q) \in D^{T}, t \in I(z) \backslash J_{c}$ and any direction $(r, u) \in R \times R^{2 n}$, the contingent derivatives in (2.1) of the components of the characteristic flow are related as follows:

$$
\begin{aligned}
& K^{+} V((t, z) ;(r, u))=\left\{<P(t, z), v_{1}>-r . H\left(t, X^{*}(t, z)\right)-<q, u_{1}>;\right. \\
& \left.v_{1} \in K^{+} X((t ; z) ;(r, u))\right\}, \text { if } u=\left(u_{1}, u_{2}\right), t \in I(z) \backslash J_{c}, z \in D^{T} .
\end{aligned}
$$

In particular, if $X(.,$.$) is differentiable at (t, z) \in D_{h}$ then $V(.,$.$) is also differen-$ tiable at the same point and the formula in (1.1) is verified.

Proof. We prove first that outside of the null subset $J_{c}$ in (3.2) the contingent derivatives of the characteristic flow $C^{*}(.,$.$) (and therefore, of each of its components)$ satisfy the relation:

$$
K^{+} C^{*}((t, z) ;(r, u))=D_{1} C^{*}(t, z) \cdot r+K^{+} C^{*}(t, .)(z ; u) \text { if } t \in I(z) \backslash J_{c} .
$$

To prove the inclusion " $\subseteq$ " we consider $v \in K^{+} C^{*}((t, z) ;(r, u))$ and note that from (2.1) it follows that there exists a sequence $\left(s_{m}, r_{m}, u_{m}\right) \rightarrow\left(0_{+}, r, u\right)$ such that:

$$
v=\lim _{m \rightarrow \infty} \frac{1}{s_{m}}\left[C^{*}\left(t+s_{m} r_{m}, z+s_{m} u_{m}\right)-C(t, z)\right] ;
$$

next, since $C^{*}(t,$.$) is Lipschitzean, taking possibly a subsequence one may assume$ that:

$$
\begin{aligned}
& \exists v^{2}:=\lim _{m \rightarrow \infty} \frac{1}{s_{m}}\left[C^{*}\left(t, z+s_{m} u_{m}\right)-C^{*}(t, z)\right] \in K^{+} C^{*}(t, .)(z ; u) \\
& v-v^{2}=v^{1}:=\lim _{m \rightarrow \infty} \frac{1}{s_{m}}\left[C^{*}\left(t+s_{m} r_{m}, z+s_{m} u_{m}\right)-C\left(t, z+s_{m} u_{m}\right)\right] .
\end{aligned}
$$


Using the "integrable-Lipschitz" property in Def.2.3 of $c(.,$.$) and the "usual" Lipschitz$ property in Th.3.2 of $C^{*}(.,$.$) one may easily prove that:$

$$
\begin{aligned}
& v^{1}:=\lim _{m \rightarrow \infty} \frac{1}{s_{m}} \int_{t}^{t+s_{m} r_{m}} c\left(s, X^{*}\left(s, z+s_{m} u_{m}\right)\right) d s= \\
& =\lim _{m \rightarrow \infty} \frac{1}{s_{m}} \int_{t}^{t+s_{m} r_{m}} c\left(s, X^{*}(s, z)\right) d s=D_{1} C^{*}(t, z) \cdot r
\end{aligned}
$$

(since $\left.t \in I(z) \backslash J_{c}\right)$ and the first inclusion in (3.19) is proved; the reversed inclusion follows in the same way noting that if $v^{2} \in K^{+} C^{*}(t,).(z ; u)$ is of the form in (3.21) and $v^{1}:=D_{1} C^{*}(t, z) \cdot r$ then using (3.22) it follows that $v:=v^{1}+v^{2}$ is of the form in (3.20) and (3.19) is proved; the relation in (3.18) is an obvious consequence of (3.7) and (3.19) and Cor.3.5 is completely proved.

We note that, apparently, the most general case in which (3.18) is verified also at the points $t \in J_{c}$ seems to be that in which the mappings $H(., z), h(., z), z \in p r_{2} D$ are regular, having one-sided limits at each point hence an at most countable number of discontinuity points, all of the first kind.

\section{References}

[1] Aubin, J. P. and H. Frankowska. (1990). Set Valued Analysis, Boston, Birkhäuser.

[2] Blagodatskih, V. I. (1973). On differentiability of solutions with respect to initial conditions, Diff. Uravn., 9, 2136-2140 (in Russian).

[3] Courant, R. (1962). Partial Differential Equations, N.Y., Interscience.

[4] Hartman, Ph. (1964) Ordinary Differential Equations, N.Y., Wiley

[5] Kurzweil, J. (1986) Ordinary Differential Equations, Amsterdam, Elsevier,.

[6] Mirică, Şt. (1982). The contingent and the paratingent as generalized derivatives of vector-valued and set-valued mappings, Nonlinear Anal., Theory, Meth. Appl., 6, 1335-1368.

[7] Mirică, Şt. (1985).On some generalizations of the Bendixson-Picard theorem in the theory of differential equations, Bull. Math. Soc. Sci. Math. Roumanie, 29(77), 315-328.

[8] Mirică, Şt. (1987).Generalized solutions by Cauchy's Method of Characteristics, Rend. Sem. Mat. Univ. Padova, 77, 317-350.

[9] Mirică, Şt. (1995). Quasitangent differentiability with respect to initial data for Carathéodory-Lipschitz differential equations, in "Qualitative problems for Differential Equations and Control Theory", C. Corduneanu Ed., New Jersey, Singapore, World Scientific, 81-89.

[10] Mirică, Şt. (2002). On differentiability with respect to initial data in the theory of Differential Equations, Revue Roum. Math. Pure Appl., submitted.

[11] Mirică, Şt. and C. Neculăescu (1998). On a semi-smooth Hamiltonian system and related Hamilton-Jacobi equations, Anal. Univ. "Ovidius", Constanţa, Ser. Mat. 6.

[12] Scorza-Dragoni, G. (1948). Una teorema sulla funzione continue rispètto ad una e misurabile rispètto ad un'altra variabile, Rend. Sem. Mat. Univ. Padova, XVII, 102-106. 